Bull. Mater. Sci., Vol. 8, No. 2, May 1986, pp. 239-246. (C) Printed in India.

\title{
Modern computer assisted methods in metallurgy
}

\author{
G W JENKINSON and T KELLY \\ Cambridge Instruments Limited, Rustat Road, Cambridge, England
}

\begin{abstract}
Image analysis systems have been used in metallurgy for over two decades and play a vital role in quality assessment for the steel industry. One major application is the characterization of steel by counting and measuring the non-metallic inclusion content. Visual steel assessment, relying on the comparison with idealized 'charts' of varying inclusion content, for example as defined by ASTM and SEP, is the most common manual method employed.

However, this has proved to be most difficult to automate due to the degree of subjectivity associated with the manual methods. This has previously restricted the acceptance of image analysis systems for routine quality control. The increased software power of the more modern instruments has enabled the installation of systems which satisfactorily mimic the skilled observer in a reproducible manner. These work with an economically attractive throughput and produce results in the accepted standard format.

A limitation of these systems is that they grade a field on the basis of the majority inclusion type present. This can lead to significant but lesser inclusions being ignored altogether and presenting a false picture of the sample. This paper describes a new method which separately identifies individual inclusions in a field. The grade number of a field can then be determined for each inclusion type. This is known as the mixed field method. This paper describes this method and its implementation on the Cambridge Instruments" QUANTIMET 920 image analysis system.
\end{abstract}

Keywords. System operation; inclusion type; inclusion grade; mixed fields; worst fields.

\section{Introduction}

The mechanical properties of steel are greatly influenced by the type and dispersion of non-metallic inclusions, and the inclusions themselves are often characteristic of the steel making practice.

The evaluation and description of the inclusion content of steel is carried out on a routine basis in every quality monitoring laboratory in the steel industry, and for economic and historical reasons, visual chart comparison procedures such as the ASTM E45 method (American Society for Testing and Materials, 1963) and the sép 1570-1 method (Stahl-Eisen-Prufblatt 1971), are frequently used. Despite their shortcomings (Johansson 1974) these chart-based methods do provide a common ground for intercompany and manufacturer-user dialogue but the precision of the results obtained by the visual methods is often far from satisfactory. Repeat evaluations by a single operator, and evaluations obtained by different operators examining the same sample, show wide variations, and to such a degree that it can be difficult to draw genuine conclusions concerning assessment carried out at different times and places by different operators (Johansson 1974). This is precisely the effect that the visual method is supposed to avoid. Automatic image analyzers such as the Cambridge Quantimet systems set out to minimize this effect by providing a standardized operation procedure for the evaluation of the inclusion fields, so that results are independent of where the evaluation is performed or who operates the system. 
It has been shown (Pohl 1979a, b) that the same measurements can be obtained by automatic image analysis as by visual comparison for the assessment of non-metallic inclusions. The first analyzer to do this for ASTM E45 and SEP 1570-71 was the Quantimet 720 Steelscan (Polzin 1977, 1978). Since then, however, more rigorous application of the standards has called for the ability to determine the content of each type of oxide in a field rather than to classify a field on the basis of the most likely single inclusion type. This is a significantly more difficult function and this paper describes the methods used and illustrates the implementation on the Quantimet 920 image analyser.

\section{General description of system operation}

A conventionally prepared specimen either mounted in plastic or as a free block, in accordance with local practice, is scanned automatically by a motorized XY stage under a microscope equipped with a high-speed autofocus system. There are few limitations on specimen size; samples up to $10 \mathrm{~cm} \times 10 \mathrm{~cm}$ can be accommodated though a total inspection area of 250 or $500 \mathrm{~mm}^{2}$ is typical. Specimens are examined under the conditions prescribed in the chart method $(\times 100)$ so the images are familiar to the operator and this allows direct confirmation of the system interpretation of field ratings.

The optical image is presented to a high resolution ( 720 line) plumbicon scanner, necessary to allow correct separation of the sulphide and oxide inclusions at the relatively low magnifications involved. The parameters required for the evaluation of inclusion type and rating are measured and the decoding of this quantitative data into the familiar chart terms is carried out by the systems' microprocessor. On completion of an analysis, the traditional evaluation table is printed (table 1 shows an ASTM example) and if required the 'worst fields' of any inclusion type can be recalled, displayed and rejected if necessary.

\section{Determination of inclusion type}

The primary classification into oxide and sulphide inclusion types is performed by an automatic detector which is sensitive to the grey tone differences of these two major inclusion types. It duplicates therefore the discrimination process of a human operator but includes fine detail enhancement to ensure that all visually recognised inclusions are identified to the system. The detection system also compensates for variations in background illumination generated in all optical microscopes and whose presence could severely limit the useful field of measurement. The separation of the oxide and sulphide inclusion types is therefore a straightforward process and is illustrated in figure 1 .

The next requirement, the classification of the oxide inclusion into the three visual types (recognized as B, C, D in the ASTM procedure and OA, OS, OG in the sEP method) is more complex. The essential differences between the three oxide types are of morphology and patternness as can be seen from the schematic representation of the chart fields in figure 2 . The silicate type is characterized by having an elongated morphology relative to the alumina and globular types which are morphologically very similar. There is a very different patternness for the alumina and globular types which 
Table 1. Typical ASTM E45 chart print-out

\begin{tabular}{|c|c|c|c|c|c|c|c|c|}
\hline \multirow[b]{2}{*}{ Grade } & \multicolumn{2}{|c|}{ Sulphides } & \multicolumn{2}{|c|}{ Aluminates } & \multicolumn{2}{|c|}{ Silicates } & \multicolumn{2}{|c|}{ Globular } \\
\hline & Thin & Thick & Thin & Thick & Thin & Thick & Thin & Thick \\
\hline \multicolumn{9}{|c|}{ Based on observed specimen area of $199.091 \mathrm{~mm}^{2}$} \\
\hline 0.5 & 121 & 59 & 6 & 0 & 115 & 40 & 108 & 118 \\
\hline 10 & 91 & 102 & 0 & 1 & 34 & 12 & 0 & 168 \\
\hline 1.5 & 7 & 22 & 1 & 0 & 2 & 38 & 0 & 18 \\
\hline $2 \cdot 0$ & 0 & 142 & 0 & 2 & 0 & 137 & 0 & 0 \\
\hline $2 \cdot 5$ & 0 & 8 & 0 & 3 & 0 & 3 & 0 & 0 \\
\hline $3 \cdot 0$ & 0 & 2 & 0 & $\mathbf{0}$ & 0 & 1 & 0 & 0 \\
\hline $3 \cdot 5$ & 0 & 0 & 0 & 0 & 0 & $\mathbf{0}$ & 0 & 0 \\
\hline $4 \cdot 0$ & 0 & 0 & 0 & 0 & 0 & 0 & 0 & 0 \\
\hline $4 \cdot 5$ & 0 & 0 & 0 & 0 & 0 & 2 & 0 & 0 \\
\hline $5 \cdot 0$ & 0 & 0 & 0 & 0 & 0 & 0 & 0 & 0 \\
\hline \multicolumn{9}{|c|}{ Based on standard specimen area of $250 \mathrm{~mm}^{2}$} \\
\hline 0.5 & 152 & 74 & 8 & 0 & 144 & 50 & 136 & 148 \\
\hline 1.0 & 114 & 128 & 0 & 1 & 43 & 15 & 0 & 211 \\
\hline 1.5 & 9 & 28 & 1 & 0 & 3 & 48 & 0 & 23 \\
\hline $2 \cdot 0$ & 0 & 178 & 0 & 3 & 0 & 172 & 0 & 0 \\
\hline $2 \cdot 5$ & 0 & 10 & 0 & 4 & 0 & 4 & 0 & 0 \\
\hline 3.0 & 0 & 3 & 0 & 0 & 0 & 1 & 0 & 0 \\
\hline 3.5 & 0 & 0 & 0 & 0 & 0 & 0 & 0 & 0 \\
\hline $4 \cdot 0$ & 0 & 0 & 0 & 0 & 0 & 0 & 0 & 0 \\
\hline $4 \cdot 5$ & 0 & 0 & 0 & 0 & 0 & 3 & 0 & 0 \\
\hline 5.0 & 0 & 0 & 0 & 0 & 0 & 0 & 0 & 0 \\
\hline
\end{tabular}

Number of fields rejected: 0

can be characterized by the degree of clustering along and about the direction of elongation as described by the charts.

Figure 3 describes the decision logic which classifies a given field in terms of the measurements made by the Quantimet. This logic requires the individual classification of each inclusion in the field and this is achieved by measurement of the aspect ratio, $X$ and $Y$ position and vertical length of each inclusion. The photographs of figure 4, which show identified inclusions, can be compared with figure 2 . The annotation on the photographs is performed by the system while operating in 'review mode' which allows the operator to check the decisions being made by the system. The codes used to annote the inclusions are as follows:-

$$
\begin{array}{lll}
\left.\begin{array}{l}
\text { SUL }- \text { Thick } \\
\text { sul }- \text { Thin }
\end{array}\right\} & \text { Sulphide } & \text { (A or SS) } \\
\left.\begin{array}{l}
\text { ALU }- \text { Thick } \\
\text { alu }- \text { Thin }
\end{array}\right\} & \text { Alumina oxide } & \text { (B or OA) } \\
\left.\begin{array}{l}
\text { SIL }- \text { Thick } \\
\text { sil }- \text { Thin }
\end{array}\right\} & \text { Silicate oxide } & \text { (C or OS) } \\
\left.\begin{array}{l}
\text { GLO }- \text { Thick } \\
\text { glo - Thin }
\end{array}\right\} & \text { Globular oxide } & \text { (D or OG) }
\end{array}
$$




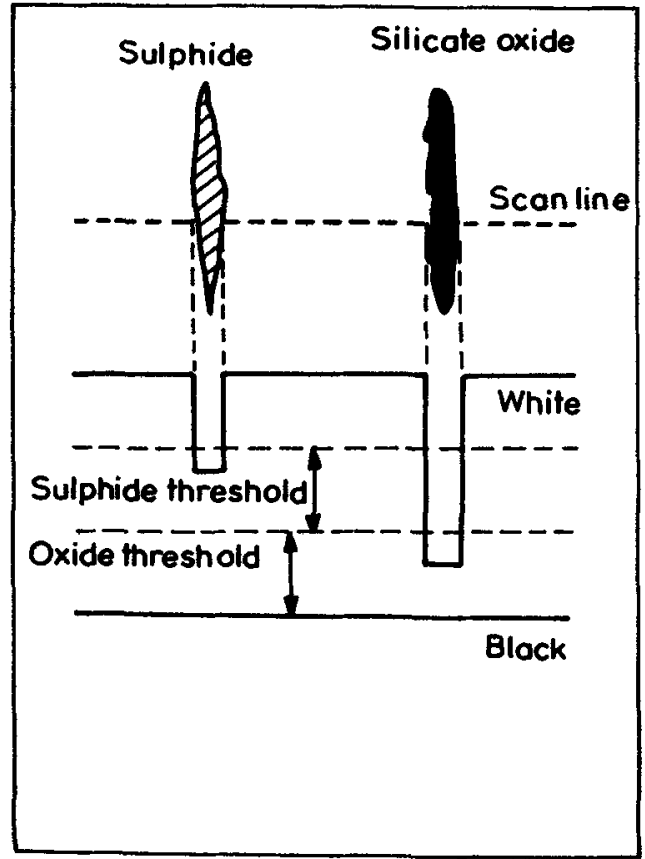

Figure 1. Separation of oxides from sulphides by grey-level

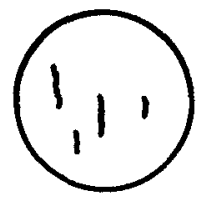

Sulphate (pale grey)

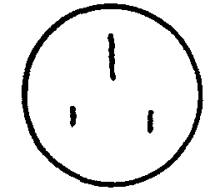

Silicate oxide

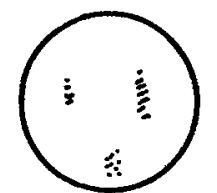

Alumina oxide

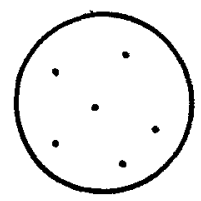

Globular-oxide

Figure 2. Schematic representation of chart fields 


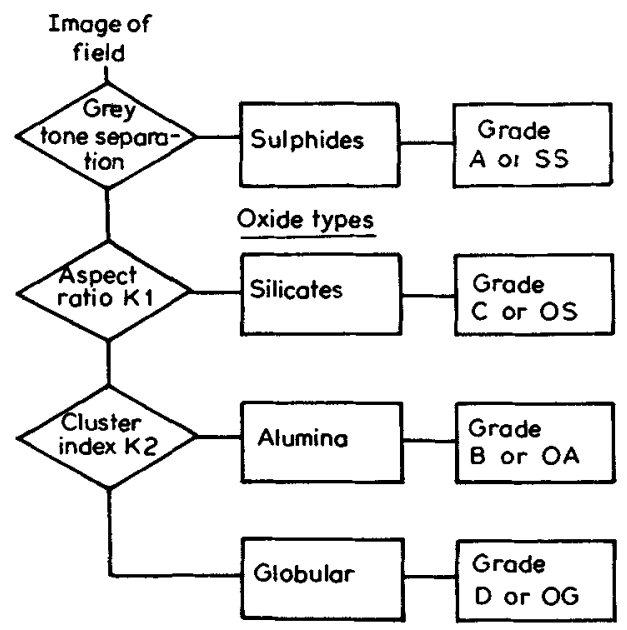

Figure 3. Classification algorithm for automatic rating: $K 1$ is a constant which is specified to separate silicates from other oxides on the basis of aspect ratio; K2 is a constant which is specified to determine the cluster spacing which separates alumina and globular oxides.

\section{Determination of inclusion grade}

Having identified the inclusion type as described, it is then a straightforward matter to assign a grade number (0-8) to the type. Each column of the ASTM Or SEP chart shows an increase in total inclusion length as we progress through the range $0-8$ and since this is a parameter measured by the Quantimet it can be used to assign a grade number to the observed field. If required the grading can be further refined into the 'thick' or 'thin' categories allowed for in the charts. The values characteristic of each ASTM or SEP grade are defined in the respective standard so these are used in the analysis programme. As can be seen from table 1 , two tables are printed. The first table shows the actual number of $0.5 \mathrm{~mm}^{2}$ fields which have been measured. The lower table has been normalized to the standard specimen area of $250 \mathrm{~mm}^{2}$. It is often preferable to scan a reduced area as this is of sufficient accuracy while improving throughput.

\section{Mixed fields}

Assigning a grade number to an observed field by comparing it with the chart representation is subjective and influenced by several factors. The occurrence of a 'severe' sulphide field will influence the operator's decision concerning the oxides in that field. Automatic image analysis gives unbiased results. The presence of 'mixed' oxide fields (alumina with globular or silicate with globular) also causes confusion.

In previous systems mixed oxide fields were not partitioned into different types and a majority field type was assigned. In the logic implemented by the Quantimet 920, each individual inclusion is identified, and its contribution to the grade number determined even for mixed oxide fields. Clearly this is a much more demanding protocol to implement and reproduction of human classification cannot be perfect. However, the provision of a review facility allows the operator to verify that the system is performing satisfactorily before making fully automatic analysis. 
$G W$ Jenkinson and $T$ Kelly
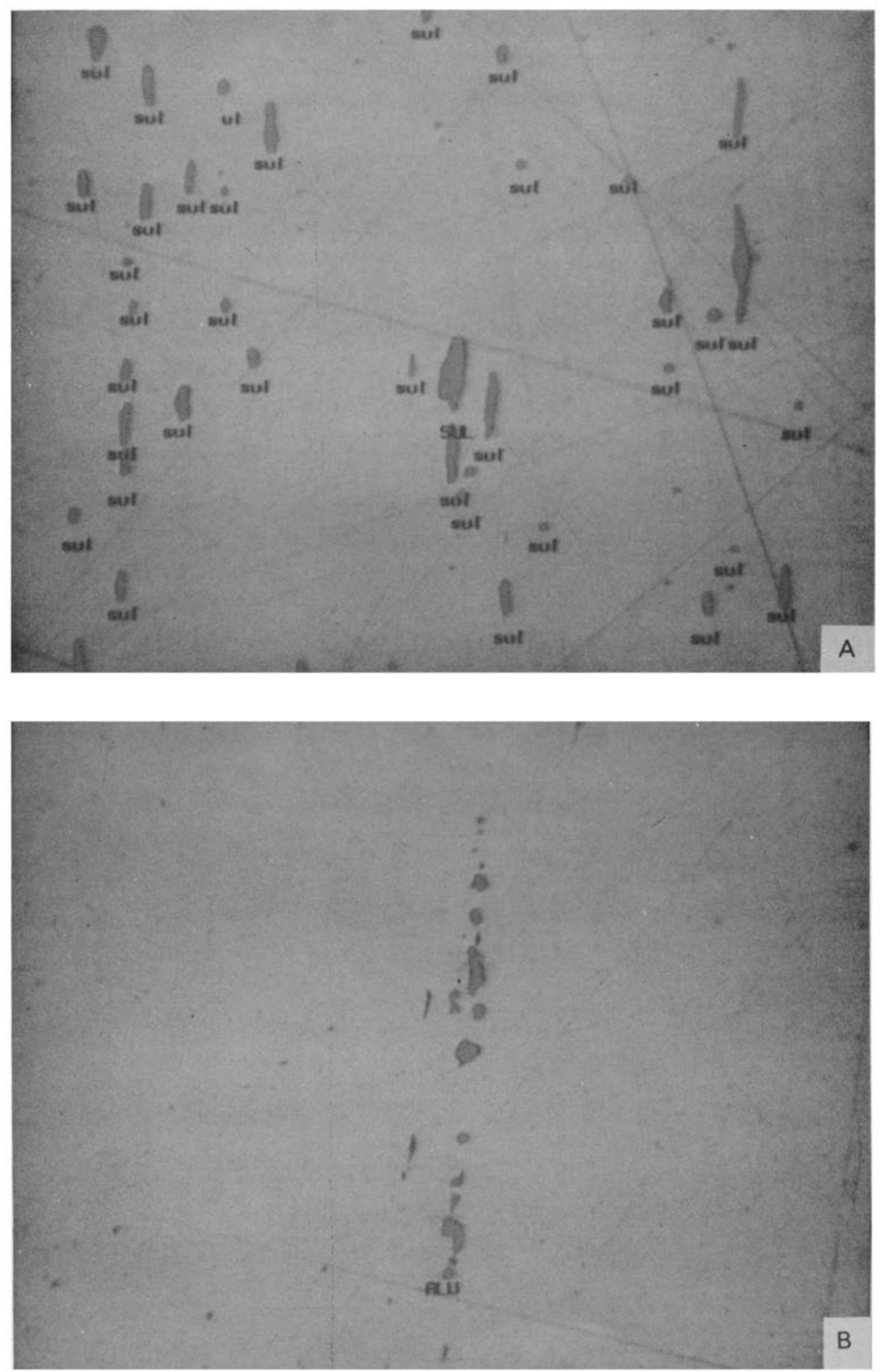

Figure 4. 


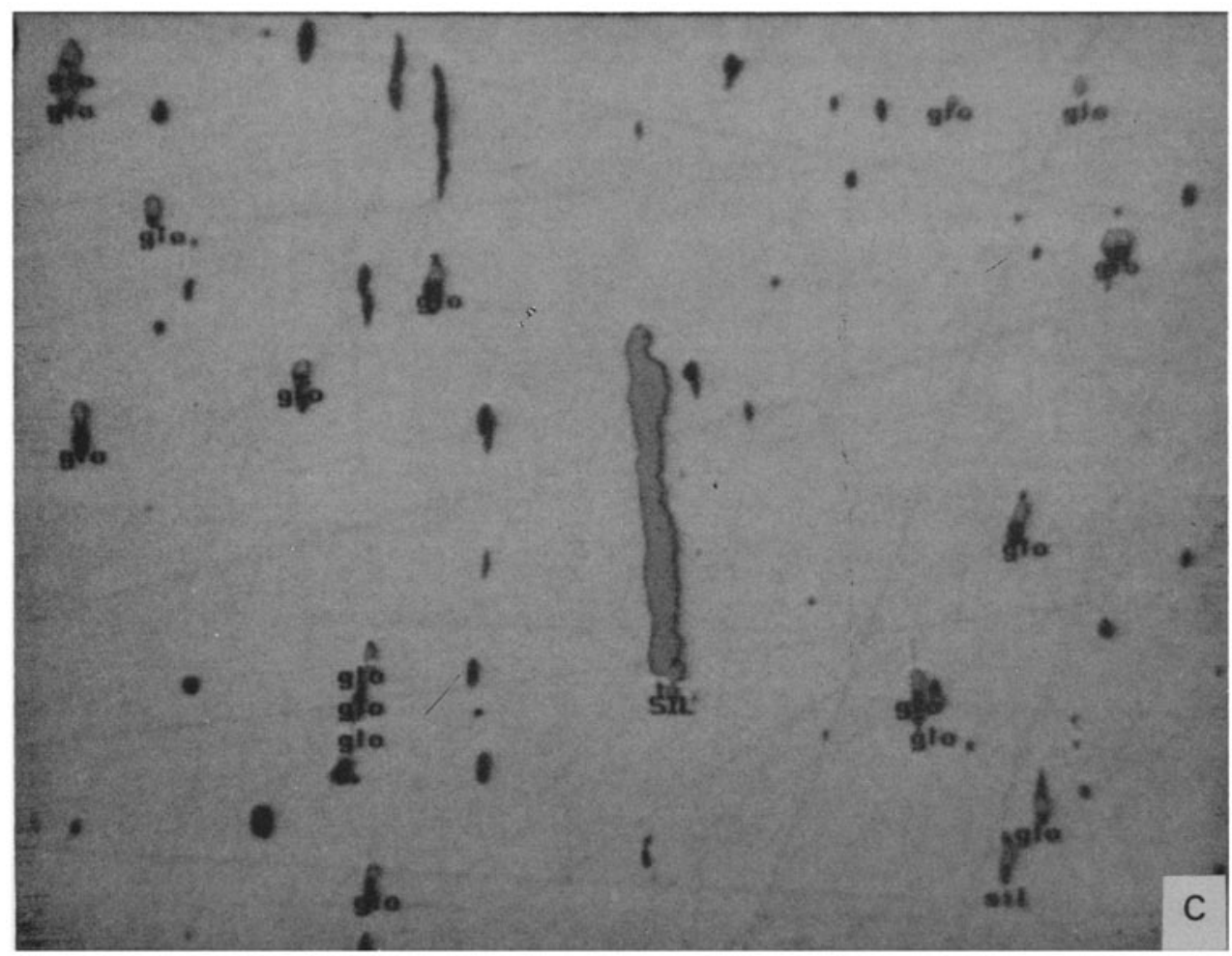

Figure 4. (A) Identified sulphide inclusions. (B) Identified alumina inclusions. (C) Identified silicate and globular inclusions.

\section{Worst fields}

Preparation methods used for automatic analysis need to be of a consistent high quality. However, dust or dirt cannot be entirely avoided. The worst field review facility enables the operator to check on the fields which have unusually high ratings. They can be excluded from the analysis if found to be due to dirt or debris.

The worst field for each inclusion type can also be recalled. The system remembers the X-Y location of the worst fields. The operator selects from the list presented (figure 5) which type of field to examine. The system moves this field of the specimen into the analysis field of view by moving the automatic motorised microscope stage. The operator can decide to accept or reject this field. The next worst field will then be presented and so on until sufficient fields have been reviewed.

\section{Conclusions}

This paper has described how the image analysis techniques for chart grading of steel cleanliness have been extended to work with mixed fields of oxides.

The implementation on the Quantimet 920 also provides for the review and rejection 


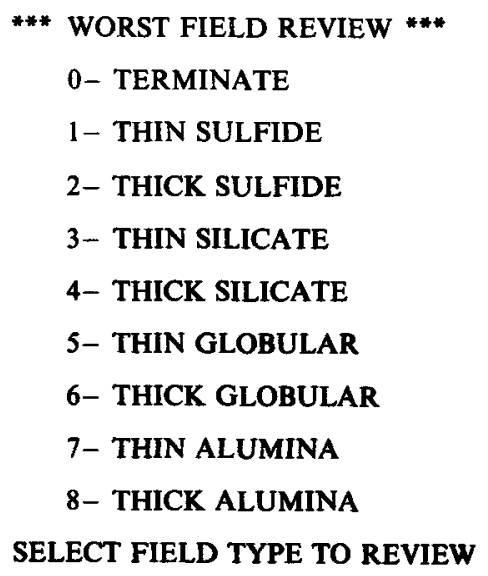

Figure 5. World field review selection.

if necessary of the worst fields found after this analysis run is complete. A verify facility permits system operation to be checked for each field.

\section{References}

ASTM 1963 American Society for Testing and Materials. "Recommended practice for determining the inclusion content of steel," ASTM E45-63

Johansson S 1974 Tech. Rept. Sandviken. "The rating of nonmetallic inclusions by image analysis."

Pohl W and Fischer A 1979a Microscopia Acta, Supplement 3, S. 77-82. "Experience in Steel Cleanliness Assessment by the Automatic Image Analyser QUANTIMET 720/10M"

Pohl W and Fischer A 1979b Sonderbande der Praktischen Metallographie, Band 10, S631-640. "Erfahrungen bei der Reinheitzgradbestimmug mit dem Automatischen Bildanalysator QUANTIMET 720/10M."

Polzin T 1977 Microscopia Acta, Supplement 1, S 19-31

Polzin T 1978 Praktische Metallographic, Band 15, S 277-286. "QUANTIMET 360/2 and QUANTIMET $720 / 10 \mathrm{M}$ - two new systems for microscopic evaluation of non-metallic inclusion content in steel."

Stahl-Eisen-Prufblatt 1971 Verlag Stahleisen, Dusseldorf. "Microscopic examination for non-metallic inclusions with reference inclusion charts." 